\title{
The return of chloroquine susceptible Plasmodium falciparum in Sub-Saharan Africa: a protocol for systematic review
}

\section{George M Bwire}

Muhimbili University of Health and Allied Sciences

\section{Ritah Mutagonda}

Muhimbili University of Health and Allied Sciences

Amisa Tindamanyile

Muhimbili University of Health and Allied Sciences

Tosi Mwakyandile

Muhimbili University of Health and Allied Sciences

Belinda Jackson Njiro ( $\sim$ bellyjacky25@gmail.com )

Muhimbili University of Health and Allied Sciences School of Medicine https://orcid.org/0000-00028312-0643

\section{Deodatus Sabas}

Muhimbili University of Health and Allied Sciences

\section{Protocol}

Keywords: Chloroquine, Plasmodium falciparum, Systematic review protocol

Posted Date: May 11th, 2020

DOI: https://doi.org/10.21203/rs.3.rs-26925/v1

License: (9) This work is licensed under a Creative Commons Attribution 4.0 International License. Read Full License 


\section{Abstract}

Background: Following withdrawal and/or restricted use of chloroquine (CQ) as first line malaria treatment in many countries, studies have reported an increased number of CQ susceptible Plasmodium falciparum in several countries with subsequent dropping of $\mathrm{CQ}$ resistance. Since the future of malaria control and elimination is still uncertain with rising resistance in currently available antimalarials such as artemisinin based combination therapy (ACT), a review on current resistance profile of $C Q$ in $P$. falciparum is of paramount importance.

Methods: A systematic search in PubMed/MEDLINE, EMBASE, COCHRANE central, Google Scholar and Web of science will be done. We will consult thesis repositories to identify additional studies and search the websites of key healthcare organizations (World Health Organization (WHO), public health agencies). Similarly, a grey literature search will be done with help of Google. Data from 2000 and onwards published in English will be included and the reporting will be done in accordance with the Preferred Reporting Items for Systematic Reviews and Meta- Analyses Protocol (PRISMA-P).

Discussion: This article provides a detailed account of this systematic review protocol that will be used to report the current status of CQ resistance in $P$. falciparum following its restricted use and/ or withdrawal as the first-line treatment of uncomplicated malaria. Mutations in $P$. falciparum chloroquine transporter ( $p f c r t$ ) gene and $P$. falciparum multidrug resistance ( $p f m d r 1$ ) gene, predict the clinical outcomes following treatment using CQ. On the other hand, countries with restricted use of CQ observed CQ-susceptible $P$. falciparum reemergence and are now predominant. Subject to its susceptibility profile that will be generated from this review, CQ may still be considered for malaria prevention in some population groups such as children with sickle cell disease and pregnant women. Additionally, CQ may be reintroduced in future, ideally in combination with other antimalarial drugs, especially in areas where disappearance of chloroquine resistance is evident while safe and affordable alternatives antimalarials are limited.

Systematic review registration: PROSPERO registration number CRD42020154844

\section{Background}

Malaria is one of the significant global health concerns. In 2017 globally, there were a total of 239 million cases with Africa and South-east Asia being highly burdened contributing $92 \%$ and $5 \%$ of the cases respectively. Additionally, malaria resulted into a total of 235,000 deaths worldwide, $61 \%$ of which were among the under-fives. Despite the global decrease in the incidence rate of malaria from 72 in 2010 to 59 in 2017 per 1000 population, the progress in malaria control and elimination is still slow especially in subSaharan Africa (1).

Plasmodium falciparum is reported to be the most predominant species causing malaria in almost all malaria endemic regions, accounting for a total of $99.2 \%$ of cases in Africa, $62.8 \%$ in South east Asia and $69 \%$ in Eastern Mediterranean (1). This explains why the mortality rate is high in these regions since falciparum species is the most dangerous of all four Plasmodium species. Climate is among several 
factors that explain the pattern of plasmodium species distribution in these regions and the mosquito Anopheles gambiae being the most efficient in malaria transmission, its lifecycle is more favored in tropical climates.

Management of malaria in endemic regions where $P$. falciparum has been reported to be most prevalent has gone through different phases; initially $C Q$ was reported to be the first line antimalarial drug in most of (all) malaria endemic regions prior to development of CQ resistant $P$. falciparum $(2,3)$. CQ use contributed to the great effort in malaria control starting from 1950's (4).

Wide use of CQ especially with presumptive treatment and mass drug administration in most countries is said to have contributed to its resistance starting from late 1950s in South-east Asia and South America and later in Africa, with pfcrt ( $P$. falciparum chloroquine transporter ) gene mutation being implicated as the biological cause (5-7). Development of $C Q$ resistant species led to the increased malarial transmission as well as morbidity and mortality (8). After the phase out of CQ most countries then adopted the use of sulfonamide-pyrimethamine alone or in combination. Currently, most of malaria endemic regions have included artemisinin based combination therapy (ACT) as the first drug of choice for management of uncomplicated malaria as recommended by WHO since 2007 (9-12).

Following withdrawal of $\mathrm{CQ}$ use as the first line antimalarial drug in many countries, studies have reported increasing number of $C Q$ susceptible species in several countries with subsequent dropping of CQ resistance with up to $>90 \%$ susceptibility were reported $(4,13-15)$. Considering more than a decade of ACT use, currently ACT has been reported to develop resistance both partial and complete in several occasions and a change of malaria treatment policy is indicated if the failure rate is $>10 \%$ (12). The future of malaria control is still uncertain with such rising ACT resistance, furthermore a review on CQ susceptible $P$. falciparum is of paramount importance. This review will form basis for considering CQ reintroduction in future, ideally in combination with other antimalarial drugs.

\section{Methods}

\section{Study registration}

The present review protocol is being reported in accordance with the reporting guidance provided in the Pre-ferred Reporting Items for Systematic Reviews and Meta- Analyses Protocols (PRISMA-P) (see PRISMA-P checklist in Additional file 1). This review protocol has been registered within the International Prospective Register of Systematic Reviews (PROSPERO database registration number:

CRD42020154844).

\section{Eligibility criteria}

\section{Type of studies and participants}

Both clinical trials and observational studies (including cross sectional, cohort and case-control studies) will be included. P. falciparum with confirmed chloroquine resistant or susceptible molecular markers. 
Several markers associated with $P$. falciparum antimalarial drug resistance have been identified (16). These include encoding chloroquine (CQ) resistance transporter ( $p f c r t$ ) and multidrug resistance transporter-1 ( $p f m d r-1$ ) both located on the food vacuole of the parasite involved in CQ resistance (17). However, the CQ transporter pfcrt is a stronger predictor of CQ resistance than $p f m d r 1$ (18). Single nucleotide polymorphisms of $p f c r t(K 76 T)$ in field isolates correlate with a resistance phenotype in in vitro assays and are sensitive markers for treatment failure in patients $(19,20)$. The single K76 is for susceptible allele, single 76T is a marker for resistant allele while $\mathrm{K} 76 / 76 \mathrm{~T}$ (susceptible/resistant) is for mixed alleles (13).

\section{Types of interventions and outcomes}

Countries with restricted use and/or withdrawal of CQ for treatment of malaria infection. Following withdrawal of CQ use as first line malaria treatment, studies have reported increasing number of CQ susceptible species in several countries with subsequent dropping of CQ resistance $(4,13,14,21)$. Invitro prevalence of chloroquine resistant $P$. falciparum and/ or prevalence of chloroquine sensitive $P$. falciparum using pfcrt and pfmdr1 molecular markers.

\section{Searches}

We will systematically search PubMed/MEDLINE, EMBASE, COCHRANE central, Google Scholar and Web of science. We will consult thesis repositories to identify additional studies and search the websites of key healthcare organizations (WHO, public health agencies). Similarly, a grey literature search will be done with help of Google. Data from 2000 and onwards published in English will be included and searches will be re-run prior to the final analysis.

\section{URL to search strategy}

We will develop a rigorous systematic search strategy with a health sciences librarian who has systematic review experience using published guidelines of the Cochrane Collaboration. The strategy will be developed for PubMed/MEDLINE (Additional File 2) and EMBASE using keywords and MeSH (MEDLINE) or EMTREE (EMBASE). To be as inclusive as possible, we will limit the search strategy to terms covering the susceptible OR chloroquine resistant $P$. falciparum. Keywords such as $p f c r t, p f m d r 1$, chloroquine, resistant, susceptible and $P$. falciparum will be used. This search strategy will also be adapted to the other databases.

\section{Data extraction (selection and coding)}

Study selection: Study selection will be managed using Covidence software (Australia) where two independent reviewers will evaluate articles for potential inclusion by screening titles and abstracts and will assess full publications to determine eligibility for final inclusion. Between each assessment, results will be discussed to reach a consensus on the interpretation of inclusion criteria. Any further disagreement on study eligibility will be resolved by consensus, and a third reviewer will be consulted if necessary. If information on eligibility is unavailable and/ or unclear, study authors will be contacted to 
clarify. Duplicate publications will be identified and removed using reference manager (Endnote X9, USA). Identified publication(s) will be analyzed using criteria based on most recent dates, largest sample size, maximum correspondence with inclusion criteria and minimal risk of bias.

Data coding: Data that will be extracted from study documents, including information about study design and methodology, participant demographics and baseline characteristics, prevalence of chloroquine resistant/ susceptible etc (Additional File 3). Unavailable, unclear information and/or additional details will be requested from the study investigators. Data will be recorded using excel spreadsheet and Systematic Review Data Repository-Plus.

\section{Risk of bias (quality) assessment}

Risk of bias in observational studies will be evaluated using the ROBINS-I risk of bias assessment tool for non-randomized studies. The tool evaluates baseline and time-varying confounding, co-interventions, selection bias, classification bias (intervention), missing data, and bias in outcome measurement. While for randomized controlled trials risk of bias will be evaluated using the Cochrane risk of bias tool. Two reviewers will independently evaluate risk of bias and rate studies using respective tools (low, moderate serious, critical, unclear for the ROBINS-I tool and high, low, unclear for the Cochrane risk of bias tool). Disagreement will be resolved using arbitration by a third reviewer

\section{Strategy for data synthesis}

Depending on data availability the trend of chloroquine resistance will be sorted per year based on change of chloroquine treatment. For heterogeneity across studies in terms of populations, design, methods, intervention, and/or outcome (s) will be presented using a systematic narrative synthesis. We will explore the results according to categories of interventions and outcomes taking account of risk of bias, in line with Centre for Reviews and Dissemination recommendations. The narrative will be written by the lead reviewer and then checked independently by at least one other reviewer. A third reviewer will adjudicate any disagreements. We will measure heterogeneity for each meta-analysis using $R$.

\section{Analysis of subgroups or subsets}

When data available the trend of chloroquine resistance will be sorted based on year of abandonment in every country.

\section{Discussion}

This article provides a detailed account of this systematic review protocol that will be used to report the current status of CQ resistance in $P$. falciparum following its restricted and/ or withdrawal as the first-line antimalarial drug for treatment of uncomplicated malaria.

Gene mutations in pfcrt and pfmdr1 as a result of pressure use of CQ, predicts CQ resistance of $P$. falciparum malaria predict the clinical outcomes following the treatment using CQ (22). Pfmdr1 
mutations do contribute to treatment failure, but they provide an assistance to pfcrt mutations to exert an effect on the response to chloroquine. However, persistence of pfmdr1 mutations in a population with a very low prevalence of pfcrt mutations would not be expected to reduce CQ efficacy in that population.

On the other hand, countries with restricted used of CQ observed CQ-susceptible $P$. falciparum parasites have reemerged and are now predominant $(4,13,14,23)$. In contrary to this, high levels of CQ resistance have persisted in countries with unrestricted use of CQ, i.e., Nigeria (24). This observation suggests that continual circulation of CQ in the study area increase spread of CQ-resistant $P$. falciparum parasites (24). The suggested cause of high levels of CQ resistance in Nigeria concurred with an evidence which documented that, the withdrawal of antimicrobial drug pressure does not always compromise the fitness of resistant microorganisms and result in selection of drug-sensitive phenotypes (25).

Lastly, after the ban of CQ use in treatment of uncomplicated malaria, the drug was still recommended for use as chemoprophylaxis against malaria in sickle cell disease patients in sub-Saharan countries including Tanzania (26) but pressure from the drug resistance stopped its use as chemoprophylaxis (27). Subject to its susceptibility profile that will be generated from this review, chloroquine may still be considered for malaria prevention, i.e. sickle cell disease children, intermittent preventive therapy in pregnancy women or the reintroduction in future, ideally in combination with other antimalarial drugs (28), especially in areas where disappearance of chloroquine resistance is evident while safe and affordable alternatives antimalarials are limited.

\section{Abbreviations}

ACT: Artimesinin based combination therapy

CQ: Chloroquine

pfcrt. Plasmodium falciparum chloroquine transporter

pfmdr1: Plasmodium falciparum

PRISMA-P: Preferred Reporting Items for Systematic Reviews and Meta-Analyses Protocols

PROSPERO: Prospective Register of Systematic Reviews

WHO: World Health Organization

\section{Declarations}

\section{Ethics approval and consent to participate}

No ethics approval is required for this systematic review because information will be extracted from published studies (secondary sources). 


\section{Consent for publication}

Not applicable.

\section{Availability of data and materials}

Not applicable.

\section{Competing interests}

The authors declare that they have no competing interests.

\section{Funding}

This work was supported by the Education Foundation of Chengdu University of Traditional Chinese Medicine.

\section{Authors' contributions}

GMB and BJN designed the study protocol and drafted the manuscript. TM, AT, RM and DS revised the manuscript. All authors have read and approved the final version of this manuscript

\section{Acknowledgements}

Authors acknowledge the training support received from Muhimbili University of Health and Allied Sciences through systematic review and meta-analysis workshop.

\section{References}

1. WHO. World Malaria Report 2018 [Internet]. 2018. Available from: https://www.who.int/malaria/publications/world-malaria-report-2018/en/

2. Elena R, Torres M, Banegas El, Mendoza M, Diaz C, Tamara S, et al. Efficacy of Chloroquine for the Treatment of Uncomplicated Plasmodium falciparum Malaria in Honduras. 2013;88(5):850-4.

3. Frosch AEP, Venkatesan M, Laufer MK. Patterns of chloroquine use and resistance in sub-Saharan Africa: a systematic review of household survey and molecular data. 2011;1-10.

4. Laufer MK, Takala-Harrison S, Dzinjalamala FK, Stine OC, Taylor TE, Plowe CV. Return of Chloroquine-Susceptible Falciparum Malaria in Malawi Was a Reexpansion of Diverse Susceptible Parasites. J Infect Dis. 2010;202(5):801-8.

5. Amar Bir Singh Sidhu, Dominik Verdier-Pinard and DAF. Chloroquine Resistance in Plasmodium falciparum Malaria Parasites Conferred by pfcrt Mutations. Science (80- ). 2010;298(5591):210-3.

6. Bloland PB. Drug resistance in malaria. World Heal Organ. 2001;WHO/CDS/CS.

7. Laufer MK, Plowe C V. Withdrawing antimalarial drugs: Impact on parasite resistance and implications for malaria treatment policies. Drug Resist Updat. 2004;7(4-5):279-88. 
8. Nuwaha F. Review article The challenge of chloroquine-resistant malaria in sub-Saharan. 2001;16(1):1-12.

9. Watsierah CA, Ouma C. Access to artemisinin-based combination therapy ( ACT ) and quinine in malaria holoendemic regions of western Kenya. 2014;13(1):1-7.

10. Aaa $\mathrm{O}, \mathrm{Cl}$ G, Garner P. Artemether-lumefantrine for treating uncomplicated falciparum malaria ( Review ). 2019;(2).

11. Treatment FORTHE, Malaria OF. Guidelines For the treatment of malaria. World Heal Organ. 2012; Third Edit.

12. Subregion GM. Artemisinin resistance and artemisinin-based combination therapy efficacy. 2018; (August).

13. Mohammed A, Ndaro A, Kalinga A, Manjurano A, Mosha JF, Mosha DF, et al. Trends in chloroquine resistance marker , Pfcrt-K76T mutation ten years after chloroquine withdrawal in Tanzania. Malar J [Internet]. 2013;12(1):1. Available from: Malaria Journal

14. Mwanza S, Joshi S, Nambozi M, Chileshe J, Malunga P, Kabuya JBB, et al. The return of chloroquinesusceptible Plasmodium falciparum malaria in Zambia. Malar J. 2016;15(1):1-6.

15. Kublin JG, Cortese JF, Njunju M, Mukadam RAG, Wirima JJ, Kazembe PN, et al. Reemergence of Chloroquine-Sensitive Plasmodium falciparum Malaria after Cessation of Chloroquine Use in Malawi. 2003;187:1-6.

16. Alifrangis M, Lusingu JP, Mmbando B, Dalgaard MB, Vestergaard LS, Ishengoma D, et al. Five-year surveillance of molecular markers of Plasmodium falciparum antimalarial drug resistance in Korogwe District, Tanzania: accumulation of the $581 \mathrm{G}$ mutation in the P. falciparum dihydropteroate synthase gene. Am J Trop Med Hyg. 2009;80.

17. H. Z, M. P, P.D. R. The antimalarial drug resistance protein Plasmodium falciparum chloroquine resistance transporter binds chloroquine. Biochemistry [Internet]. 2004;43(26):8290-6. Available from: http://www.embase.com/search/results? subaction=viewrecord\&from=export\&id=L38902508\%0Ahttp://dx.doi.org/10.1021/bi049137i

18. Pulcini S, Staines HM, Lee AH, Shafik SH, Bouyer G, Moore CM, et al. Mutations in the Plasmodium falciparum chloroquine resistance transporter, PfCRT, enlarge the parasite's food vacuole and alter drug sensitivities. Sci Rep [Internet]. 2015;5(August):1-16. Available from: http://dx.doi.org/10.1038/srep14552

19. Picot S, Olliaro P, De Monbrison F, Bienvenu AL, Price RN, Ringwald P. A systematic review and metaanalysis of evidence for correlation between molecular markers of parasite resistance and treatment outcome in falciparum malaria. Malar J. 2009;8(1):1-15.

20. Ecker A, Lehane AM, Clain J, Fidock DA. PfCRT and its role in antimalarial drug resistance. Trends Parasitol. 2012;28(11):504-14.

21. Kublin JG, Cortese JF, Njunju EM, G. Mukadam RA, Wirima JJ, Kazembe PN, et al. Reemergence of Chloroquine-Sensitive Plasmodium falciparum Malaria after Cessation of Chloroquine Use in Malawi . J Infect Dis. 2003;187(12):1870-5. 
22. Plasmodium BC. a Mol Ecul Ar Marke R F or Ch Loroquine-R Es Ista Nt Fa Lcipa Rum Ma L a Ria a Molecular Marker for Chloroquine-Resistant. English J [Internet]. 2001;344(4):257-63. Available from: http://www.ncbi.nlm.nih.gov/pubmed/11172152

23. Bwire GM, Ngasala B, Mikomangwa WP, Kilonzi M, Kamuhabwa AAR. Detection of mutations associated with artemisinin resistance at k13-propeller gene and a near complete return of chloroquine susceptible falciparum malaria in Southeast of Tanzania. Sci Rep [Internet]. 2020;10(1):1-7. Available from: http://dx.doi.org/10.1038/s41598-020-60549-7

24. Ikegbunam MN, Nkonganyi CN, Thomas BN, Esimone CO, Velavan TP, Ojurongbe O. Analysis of Plasmodium falciparum Pfcrt and Pfmdr1 genes in parasite isolates from asymptomatic individuals in Southeast Nigeria 11 years after withdrawal of chloroquine. Malar J [Internet]. 2019;18(1):1-7. Available from: https://doi.org/10.1186/s12936-019-2977-6

25. Enne VI, Livermore DM, Stephens P, Hall LMC. Persistence of sulphonamide resistance in Escherichia coli in the UK despite national prescribing restriction. Lancet. 2001;357(9265):1325-8.

26. Ndegeulaya EJ, Bwire GM, Sangeda RZ, Mloka D, Tungaraza F, Kahere AS, et al. Utilization patterns of malaria chemoprophylaxis among Tanzanian children attending sickle cell clinic in Dar es Salaam tertiary hospitals. Malar J [Internet]. 2019;10-4. Available from: https://doi.org/10.1186/s12936-0193029-y

27. Hussein Mwinyi H. the United Republic of Tanzania Standard Treatment Guidelines and Essential Medicines List Ministry of Health and Social Welfare Fourth Edition. 2013;(December):42-3. Available from: http://www.who.int/selection_medicines/country_lists/Tanzania_STG_052013.pdf

28. Krishna S. Triple artemisinin-containing combination anti-malarial treatments should be implemented now to delay the emergence of resistance: The case against. Malar J [Internet]. 2019;18(1):1-3. Available from: https://doi.org/10.1186/s12936-019-2976-7

\section{Supplementary Files}

This is a list of supplementary files associated with this preprint. Click to download.

- Additionalfile1.doc

- Additionalfile2.docx

- Additionalfile3.docx 